\title{
Investigation on Extended Number Field
}

\author{
Ma Ning a,", Li Jian-pin ${ }^{\mathrm{a}}$ \\ ${ }^{a}$ Air Force Radar Academy, Wuhan, China
}

\begin{abstract}
This paper expands the field of complex number to general plural. Discussion on the forth power general plural is emphasized, then creates the four fundamental operations of arithmetic and calculus which can be applied to the project.
\end{abstract}

Index Terms: extended number field, general plural, quartic

(C) 2012 Published by MECS Publisher. Selection and/or peer review under responsibility of the Research Association of Modern Education and Computer Science.

\section{Introduction}

Extended number field shall fall across plenty of problem, for example:definition of general plural;four fundamental operations of general plural;function's calculous in general plural field;norm axiom's problem in general plural field;Fourier transform in general plural field;engineering application's problem after extended number field.In order to expand number's extension, we can define $i^{n}=-1\left(n=2 k, k \in N^{+}\right)$. Using this definition,we can find some theoretics of general plural to expand number's extension.For example:when $\mathrm{n}=2$, that is plural we defined before. This paper emphasizes the argumentation on circs when $n=4$, and calls it quartic general plural,then strikes up four fundamental and calculous operations;morover probes deeply into the matter on this $\operatorname{circs}^{[1]}$.

Definiens for quartic general plural:

$i^{4}=-1, i^{2}=j ;$ once more $j^{2}=-1$ 。

Underside, we attempt to establish some basic operations.

\section{Discussion in four fundamental operations of forth power general plural}

Considering the geometry significance of general plural,first considers circs when $\mathrm{n}=4$, generic form is:

$z=r+a i+b j+c i j \quad(r \in R, a \in R, b \in R, c \in R, i j=i \times j)$

* Corresponding author.

| E-mail address: $156449871 @ q q . c o m$ 
Defining:several conjugate of $z$ :

$$
\begin{aligned}
& \bar{z}_{i}=r-a i+b j+c i j, \quad \bar{z}_{j}=r+a i-b j+c i j, \\
& \bar{z}_{i j}=r+a i+b j-c i j, \quad \bar{z}_{i, j}=r-a i-b j+c i j, \\
& \bar{z}_{i, i j}=r-a i+b j-c i j, \quad \bar{z}_{j, i j}=r+a i-b j-c i j, \\
& z+\bar{z}_{i, i j}=2 r+2 b j, \\
& z \times \bar{z}_{i, i j}=\left(r^{2}+2 a c-b^{2}\right)+\left(2 r b-a^{2}+c^{2}\right) j 。
\end{aligned}
$$

We can prove formula below from this definition:

$$
\overline{\bar{z}}_{k}=z_{k}(\mathrm{k}=\mathrm{i}, \mathrm{j}, \mathrm{ij},(\mathrm{i}, \mathrm{j}),(\mathrm{i}, \mathrm{ij}),(\mathrm{j}, \mathrm{ij})) ; \overline{z_{1} \pm z_{2}}=\bar{z}_{1} \pm \bar{z}_{2} ; \overline{z_{1} \times z_{2}}=\bar{z}_{1} \times \bar{z}_{2} \text { 。 }
$$

\section{Investigation on the derivation of forth power general plural}

Defining: suppose function $\omega=f(z)$ is defined in domain $\mathrm{D} 。 z_{0}$ is one point in domain $\mathrm{D}$, point $z_{0}+\Delta z$ doesn't exceed domain D。If limit $\lim _{\Delta z \rightarrow 0} \frac{f\left(z_{0}+\Delta z\right)-f\left(z_{0}\right)}{\Delta z}$ exists, well then,it is said that $f(z)$ is derivable at $z_{0}$, the limit is intituled differential coefficient of $f(z)$ at $z_{0}$, it is marked: $f^{\prime}\left(z_{0}\right)=\left.\frac{d w}{d z}\right|_{z=z_{0}}=\lim _{\Delta z \rightarrow 0} \frac{f\left(z+z_{0}\right)-f\left(z_{0}\right)}{\Delta z}$ 。

Because the differential coefficient of function of complex variable is formally same as function of real variable,and the algorithm is same too,thus the function of real variable derivation's rule can apply to the function of complex variable, for example:

(1) $\quad(c)^{\prime}=0, \mathrm{c}$ is complex constant thereinto.

(2) $\quad\left(z^{n}\right)^{\prime}=n z^{n-1}, \mathrm{n}$ is positive integer thereinto.

(3) $[f(z) \pm g(z)]^{\prime}=f^{\prime}(z) \pm g^{\prime}(z)$.

(4) $[f(z) g(z)]^{\prime}=f^{\prime}(z) g(z) \pm f(z) g^{\prime}(z)$.

Lemma 1 (forth power general plural's spread Cauchy-Riemann conditions)

$\frac{\partial a}{\partial x}=\frac{\partial b}{\partial y}=\frac{\partial c}{\partial v}=\frac{\partial d}{\partial w} ; \quad \frac{\partial b}{\partial x}=\frac{\partial c}{\partial y}=\frac{\partial d}{\partial v}=-\frac{\partial a}{\partial w} ;$

$\frac{\partial c}{\partial x}=\frac{\partial d}{\partial y}=-\frac{\partial a}{\partial v}=-\frac{\partial b}{\partial w} ; \quad \frac{\partial d}{\partial x}=-\frac{\partial a}{\partial y}=-\frac{\partial b}{\partial v}=-\frac{\partial c}{\partial w}$

Thereinto

$f(z)=a(x, y, v, w)+i b(x, y, v, w)+j c(x, y, v, w)+i j d(x, y, v, w) ; \quad z=x+i y+j v+i j w$

Attestation:if

$f(z)=a(x, y, v, w)+i b(x, y, v, w)+j c(x, y, v, w)+i j d(x, y, v, w)$ is differentiable at $z=x+i y+j v+i j w$, furthermore if

$$
\begin{aligned}
& \lim _{\Delta z \rightarrow 0} \frac{f(z+\Delta z)-f(z)}{\Delta z}=f^{\prime}(z) \\
& \Delta z=\Delta x+i \Delta y+j \Delta v+i j \Delta w ; \\
& \Delta f(x)=f(z+\Delta z)-f(z)=\Delta a+i \Delta b+j \Delta c+i j \Delta d ; \\
& \Delta a=a(x+\Delta x, y+\Delta y, v+\Delta v, w+\Delta w)-a(x, y, v, w) ; \\
& \Delta b=b(x+\Delta x, y+\Delta y, v+\Delta v, w+\Delta w)-b(x, y, v, w) ;
\end{aligned}
$$


$\Delta c=c(x+\Delta x, y+\Delta y, v+\Delta v, w+\Delta w)-c(x, y, v, w)$
$\Delta d=d(x+\Delta x, y+\Delta y, v+\Delta v, w+\Delta w)-d(x, y, v, w)$

Acquirability from formula 3.1

$$
\lim _{\Delta x \rightarrow 0, \Delta y \rightarrow 0, \Delta v \rightarrow 0, \Delta w \rightarrow 0} \frac{\Delta a+i \Delta b+j \Delta c+i j \Delta d}{\Delta x+i \Delta y+j \Delta v+i j \Delta w}=f^{\prime}(z)
$$

Because formula 3.2 is always existed no matter what direction $\Delta z=\Delta x+i \Delta y+j \Delta v+i j \Delta w$ goes to zero,so first suppose $\Delta y=\Delta v=\Delta w=0, \Delta x \rightarrow 0$, namely $z+\Delta z$ goes to point $z$ along parallelism axis $x$, here formula 3.2 becomes:

$$
\lim _{\Delta x \rightarrow 0} \frac{\Delta a}{\Delta x}+i \lim _{\Delta x \rightarrow 0} \frac{\Delta b}{\Delta x}+j \lim _{\Delta x \rightarrow 0} \frac{\Delta c}{\Delta x}+i j \lim _{\Delta x \rightarrow 0} \frac{\Delta d}{\Delta x}=f^{\prime}(z)
$$

And then $\frac{\partial a}{\partial x}, \frac{\partial b}{\partial x}, \frac{\partial c}{\partial x}, \frac{\partial d}{\partial x}$ really exists,

furthermore, $\frac{\partial a}{\partial x}+i \frac{\partial b}{\partial x}+j \frac{\partial c}{\partial x}+i j \frac{\partial d}{\partial x}=f^{\prime}(z)$

Acquirability from same principle:

$$
\begin{aligned}
& \frac{\partial b}{\partial y}+i \frac{\partial c}{\partial y}+j \frac{\partial d}{\partial y}-i j \frac{\partial a}{\partial y}=f^{\prime}(z) \\
& \frac{\partial c}{\partial v}+i \frac{\partial d}{\partial v}-j \frac{\partial a}{\partial v}-i j \frac{\partial b}{\partial v}=f^{\prime}(z) \\
& \frac{\partial d}{\partial w}-i \frac{\partial a}{\partial w}-j \frac{\partial b}{\partial w}-i j \frac{\partial c}{\partial w}=f^{\prime}(z)
\end{aligned}
$$

Lemma 1 can be achieved from formula 3.2.1 to 3.2.4.

Theorem 1(forth power general plural's differentiable necessary and sufficient condition in domain D) Suppose function $f(z)=a(x, y, v, w)+i b(x, y, v, w)+j c(x, y, v, w)+i j d(x, y, v, w)$ has definition in domain $\mathrm{D}$, then $f(z)$ 's differentiable necessary and sufficient condition in domain $\mathrm{D}$ at point $z=x+i y+j v+i j w$ is ${ }^{[2][3]}$ :

(1) quaternion function

$a(x, y, v, w), b(x, y, v, w), c(x, y, v, w), d(x, y, v, w)$ is differentiable at point $\mathrm{z}$;

(2) $a(x, y, v, w), b(x, y, v, w), c(x, y, v, w), d(x, y, v, w)$ satisfy spread Cauchy-Riemann conditions.

Thereinto one expressions is $f^{\prime}(z)=\frac{\partial a}{\partial x}+i \frac{\partial b}{\partial x}+j \frac{\partial c}{\partial x}+i j \frac{\partial d}{\partial x}$

Attestation:necessary condition:

$\Delta f(z)=f^{\prime}(z) \Delta z+o(\Delta z)$, thereinto suppose

$f^{\prime}(z)=\alpha+i \beta+j \gamma+i j \eta$

$\Delta f(z)=\Delta a+i \Delta b+j \Delta c+i j \Delta d=$

$(\alpha+i \beta+j \gamma+i j \eta)(\Delta x+i \Delta y+j \Delta v+i j \Delta w)+\rho(\Delta z) \Delta z$

Thereinto $\rho(\Delta z)=\rho_{1}+i \rho_{2}+j \rho_{3}+i j \rho_{4}, \lim _{\Delta z \rightarrow 0} \rho(\Delta z)=0, \Delta z=\Delta x+i \Delta y+j \Delta v+i j \Delta w$.

Expand formula (3.3),compare both sides of the equation,we can get

$$
\begin{aligned}
& \Delta a=\alpha \Delta x-\eta \Delta y-\gamma \Delta v-\beta \Delta w+\rho_{1} \Delta x-\rho_{4} \Delta y-\rho_{3} \Delta v-\rho_{2} \Delta w \\
& \Delta b=\beta \Delta x+\alpha \Delta y-\eta \Delta v-\gamma \Delta w+\rho_{2} \Delta x+\rho_{1} \Delta y-\rho_{4} \Delta v-\rho_{3} \Delta w \\
& \Delta c=\gamma \Delta x+\beta \Delta y+\alpha \Delta v-\eta \Delta w+\rho_{3} \Delta x+\rho_{2} \Delta y+\rho_{1} \Delta v-\rho_{4} \Delta w
\end{aligned}
$$


$\Delta d=\eta \Delta x+\gamma \Delta y+\beta \Delta v+\alpha \Delta w+\rho_{4} \Delta x+\rho_{3} \Delta y+\rho_{2} \Delta v+\rho_{1} \Delta w$

Describe it to matrix form

$$
\left[\begin{array}{c}
\Delta a \\
\Delta b \\
\Delta c \\
\Delta d
\end{array}\right]=\left[\begin{array}{cccc}
\alpha & -\eta & -\gamma & -\beta \\
\beta & \alpha & -\eta & -\gamma \\
\gamma & \beta & \alpha & -\eta \\
\eta & \gamma & \beta & \alpha
\end{array}\right]\left[\begin{array}{c}
\Delta x \\
\Delta y \\
\Delta v \\
\Delta w
\end{array}\right]+\left[\begin{array}{cccc}
\rho_{1} & -\rho_{4} & -\rho_{3} & -\rho_{2} \\
\rho_{2} & \rho_{1} & -\rho_{4} & -\rho_{3} \\
\rho_{3} & \rho_{2} & \rho_{1} & -\rho_{4} \\
\rho_{4} & \rho_{3} & \rho_{2} & \rho_{1}
\end{array}\right]\left[\begin{array}{c}
\Delta x \\
\Delta y \\
\Delta v \\
\Delta w
\end{array}\right]
$$

Owing to $\lim _{\Delta z \rightarrow 0} \rho(\Delta z)=0$, so $\lim _{\Delta z \rightarrow 0} \rho_{1}=0, \lim _{\Delta z \rightarrow 0} \rho_{2}=0, \lim _{\Delta z \rightarrow 0} \rho_{3}=0, \lim _{\Delta z \rightarrow 0} \rho_{4}=0$.

Following the differentiable definition of quaternion function, we can know

$a(x, y, v, w), b(x, y, v, w), c(x, y, v, w), d(x, y, v, w)$ is differentiable at point $\mathrm{z}$, furthermore

$\alpha=\frac{\partial a}{\partial x}=\frac{\partial b}{\partial y}=\frac{\partial c}{\partial v}=\frac{\partial d}{\partial w}$

$\beta=\frac{\partial b}{\partial x}=\frac{\partial c}{\partial y}=\frac{\partial d}{\partial v}=-\frac{\partial a}{\partial w}$

$\gamma=\frac{\partial c}{\partial x}=\frac{\partial d}{\partial y}=-\frac{\partial a}{\partial v}=-\frac{\partial b}{\partial w}$

$\eta=\frac{\partial d}{\partial x}=-\frac{\partial a}{\partial y}=-\frac{\partial b}{\partial v}=-\frac{\partial c}{\partial w}$

sufficient condition:

From condition (1), we can get:

$\Delta a=a_{x}^{\prime} \Delta x+a_{y}^{\prime} \Delta y+a_{v}^{\prime} \Delta v+a_{w}^{\prime} \Delta w$

$+\rho_{1} \Delta x-\rho_{4} \Delta y-\rho_{3} \Delta v-\rho_{2} \Delta w$

$\Delta b=b_{x}^{\prime} \Delta x+b_{y}^{\prime} \Delta y+b_{v}^{\prime} \Delta v+b_{w}^{\prime} \Delta w+\rho_{2} \Delta x+\rho_{1} \Delta y-\rho_{4} \Delta v-\rho_{3} \Delta w$

$\Delta c=c_{x}^{\prime} \Delta x+c_{y}^{\prime} \Delta y+c_{v}^{\prime} \Delta v+c_{w}^{\prime} \Delta w+\rho_{3} \Delta x+\rho_{2} \Delta y+\rho_{1} \Delta v-\rho_{4} \Delta w$

$\Delta d=d_{x}^{\prime \prime} \Delta x+d_{y}^{\prime} \Delta y+d_{v}^{\prime} \Delta v+d_{w}^{\prime} \Delta w+\rho_{4} \Delta x+\rho_{3} \Delta y+\rho_{2} \Delta v+\rho_{1} \Delta w$

Thereinto $\lim _{\Delta z \rightarrow 0} \rho_{1}=0, \lim _{\Delta z \rightarrow 0} \rho_{2}=0, \lim _{\Delta z \rightarrow 0} \rho_{3}=0, \lim _{\Delta z \rightarrow 0} \rho_{4}=0$.

From condition (2), we can get:

$\alpha=a_{x}^{\prime}=b_{y}^{\prime}=c_{v}^{\prime}=d_{w}^{\prime} ; \beta=b_{x}^{\prime}=c_{y}^{\prime}=d_{v}^{\prime}=-a_{w}^{\prime} ;$

$\gamma=c_{x}^{\prime}=d_{y}^{\prime}=-a_{v}^{\prime}=-b_{w}^{\prime} ; \quad \eta=d_{x}^{\prime}=-a_{y}^{\prime}=-b_{v}^{\prime}=-c_{w}^{\prime}$

Put equation above to

$\Delta f(x)=f(z+\Delta z)-f(z)=\Delta a+i \Delta b+j \Delta c+i j \Delta d$, we can get:

$\Delta f(x)=f(z+\Delta z)-f(z)=\Delta a+i \Delta b+j \Delta c+i j \Delta d=$

$(\alpha+i \beta+j \gamma+i j \eta)(\Delta x+i \Delta y+j \Delta v+i j \Delta w)+$

$\left(\rho_{1}+i \rho_{2}+j \rho_{3}+i j \rho_{4}\right)(\Delta x+i \Delta y+j \Delta v+i j \Delta w)$

$\frac{\Delta f(x)}{\Delta z}=\alpha+i \beta+j \gamma+i j \eta+\rho_{1}+i \rho_{2}+j \rho_{3}+i j \rho_{4}$

$\lim _{\Delta z \rightarrow 0} \frac{\Delta f(x)}{\Delta z}=\alpha+i \beta+j \gamma+i j \eta$

Namely: $f^{\prime}(x)=\alpha+i \beta+j \gamma+i j \eta$ 


\section{Investigation on Cauchy type integral of forth power general plural}

Theorem 2 if $f(z)=a(x, y, v, w)+i b(x, y, v, w)+j c(x, y, v, w)+i j d(x, y, v, w)$ is continuous along curve $\mathrm{C}$, then $f(z)$ is integrable along curve $\mathrm{C}$

$$
\begin{aligned}
& \int_{c} f(z) d z=\int_{c}(a d x-d d y-c d v-b d w)+i \int_{c}(b d x+a d y-d d v-c d w) \\
& \quad+j \int_{c}(c d x+b d y+a d v-d d w)+j \int_{c}(d d x+c d y+b d v+a d w) \quad \text { Attestation:if } z_{k}=x_{k}+i y_{k}+j v_{k}+i j w_{k}, \\
& x_{k}-x_{k-1}=\Delta x_{k}, \quad y_{k}-y_{k-1}=\Delta y_{k}, \quad v_{k}-v_{k-1}=\Delta v_{k}, \quad w_{k}-w_{k-1}=\Delta w_{k}, \quad f\left(\xi_{k}\right)=a_{k}+i b_{k}+j c_{k}+i j d_{k} \\
& \quad \text { we can get: } \\
& \quad S_{n}=\sum_{k=1}^{n} f\left(\xi_{k}\right)\left(z_{k}-z_{k-1}\right)= \\
& \sum_{k=1}^{n}\left(a_{k}+i b_{k}+j c_{k}+i j d_{k}\right)\left(\Delta x_{k}+i \Delta y_{k}+j \Delta v_{k}+i j \Delta w_{k}\right) \\
& =\sum_{k=1}^{n}\left(a_{k} \Delta x_{k}-d_{k} \Delta y_{k}-c_{k} \Delta v_{k}-b_{k} \Delta w_{k}\right)+ \\
& i \sum_{k=1}^{n}\left(b_{k} \Delta x_{k}+a_{k} \Delta y_{k}-d_{k} \Delta v_{k}-c_{k} \Delta w_{k}\right)+ \\
& j \sum_{k=1}^{n}\left(c_{k} \Delta x_{k}+b_{k} \Delta y_{k}+a_{k} \Delta v_{k}-d_{k} \Delta w_{k}\right)+ \\
& i j \sum_{k=1}^{n}\left(d_{k} \Delta x_{k}+c_{k} \Delta y_{k}+b_{k} \Delta v_{k}+a_{k} \Delta w_{k}\right)
\end{aligned}
$$

In the condition of the theorem, $a(x, y, v, w), b(x, y, v, w), c(x, y, v, w), d(x, y, v, w)$ is continuous along curve $\mathrm{C}$,then this curvilinear integral is existent,so $\int_{c} f(z) d z$ is existent, then theorem is proved.

From the integral's definition,we can detrude nether character of Cauchy type integral of general plural,they resemble function of real variable's integral character:

(1) $\int_{c} f(z) d z=-\int_{c^{-}} f(z) d z$

(2) $\int_{c} k f(z) d z=k \int_{c} f(z) d z \quad$ ( $\mathrm{k}$ 为实常数)

(3) $\int_{c}[f(z) \pm g(z)] d z=\int_{c} f(z) d z \pm \int_{c} g(z) d z$

\section{Sum-up}

Use the definition of forth power general plural to resolve several equations ${ }^{[4][5]}$ :

(1) $x^{4}+1=0$ the solution of is: $x_{x}=i ; \quad x_{2}=-i ; \quad x_{3}=i \times j ; \quad x_{4}=-i \times j$;

(2) $x^{4}+2 \times x^{2}+4 \times x+2=0$ the solution of is: $x=i+j$ 。

So the equation which is not solvable has its solution in the extended number field,this hidden solution has its use in some calculation, it can provide some new avenue to solve problem. The author is trying to use forth power general plural in engineering. 


\section{References}

[1] Philip Kitche, The Nature of Mathematical Knowledge, Oxford University Press, 1983.

[2] Yao Mu-sheng, Advanced Algebra [M]. Shanhai: Fudan University Press, 2002.

[3] Li Shizheng, Methods and Skills of Advanced Algebra [M]. Beijing: Higher Education Press, 2004.

[4] Li Wenlin, The History of Mathematics' Course [M]. Beijing: Higher Education Press, 2000.

[5] Grove, Larry C., Classical groups and geometric algebra, Graduate Studies in Mathematics, 39,

Providence, R.I.: American Mathematical Society. 2002. 